\title{
Investigations on Noise Level in AC- and DC-Bridge Circuits for Sensor Measurement Systems
}

\author{
M. A. Khan ${ }^{1}$, G. Dumstorff 1 , C. Winkelmann 1 , W. Lang ${ }^{1}$ \\ ${ }^{1}$ Institut für Mikrosensoren, -aktoren und -systeme (IMSAS), Universität Bremen, \\ Otto-Hahn-Allee NW1,28359 Bremen, Germany \\ akhan@imsas.uni-bremen.de
}

\begin{abstract}
:
Reducing noise in measurement is an important challenge in resistive sensing. This paper presents a system based on AC-excitation and lock-in amplification. In order to evaluate the circuit's ability to perform precise measurement, noise level in AC- and DC-excitation is measured. Experimental results show a promising read-out system that is capable of high noise level reduction in noisy environments. Using AC bridge excitation and lock-in amplification, the noise level is reduced by 1:55 compared with DC-excitation.
\end{abstract}

Keywords: Wheatstone Bridges, Precise measurement, AC-excitation, DC-excitation, Lock-In Amplification, Noise level reduction.

\section{Introduction}

Resistive sensors, especially strain gauges, are the workhorse of sensor technology. Like all resistors, strain gauges show thermal Nyquist noise. When a strain gauge is deformed, the piezoresistive effect causes a small change in resistivity, superimposed on a large base resistance. Using a Wheatstone bridge, the large base resistance is removed in the read out, but it is still active concerning Nyquist noise. For this reason, noise reduction is an important issue for piezoresistive sensors. In most applications, Wheatstone bridges are excited with DC voltage and read out at low frequency. As a result, there will be $1 / f$ noise (low-frequency noise) on top of Nyquist noise.

Alternatively, the excitation can be done with AC [1], avoiding $1 / f$ noise. Furthermore, ACexcitation allows lock-in amplification, reading out only a small frequency band, which allows us to remove a large part of the Nyquist noise, as well. For this investigation, a lock-in system for strain gauges is developed and applied. It is shown that noise is consequently reduced by $1: 55$.

Noise and noise suppression in resistive measurement

Fig. 1 shows a bridge circuit to measure resistivity. Noise in this system is caused by several effects.

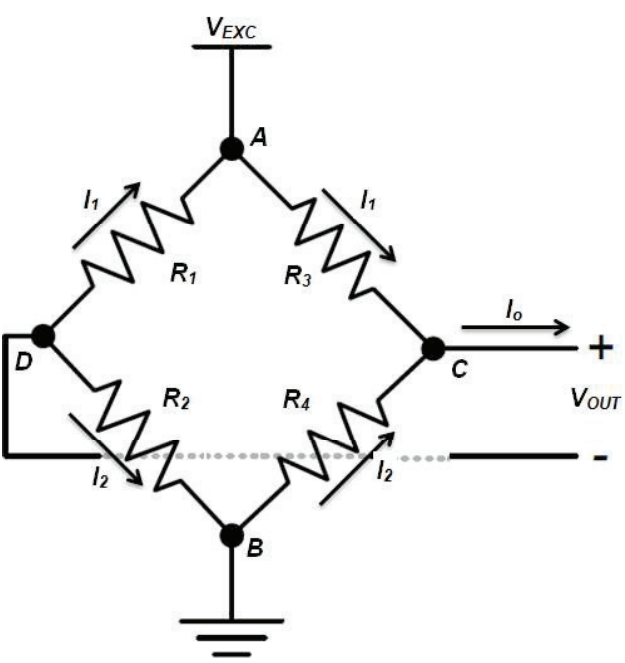

Fig.1: Wheatstone bridge circuit

Nyquist Noise (Thermal Noise)

Thermal noise places a fundamental limit on resistance measurements present in both ACexcitation and DC-excitation. Thermal energy sets particles in resistance in motion. This charge movement is called Johnson noise. In other words, it occurs through thermal agitation of the charge carriers. They are electrons inside of an electrical conductor placed in equilibrium state, which is independent of any applied voltage [2]. Such noise contains white spectrum and can be determined by temperature, resistance, and frequency bandwidth represented by:

$$
V_{\text {Nyquist }}(r m s)=\sqrt{(4 k T R B)}
$$


where

\author{
$k$ : Boltzmann's constant $\left(1,38.10^{-23} \frac{J}{K}\right)$ \\ T: Absolute Temperature in Kelvin (K) \\ R: Resistance in Ohms \\ $B$ : Noise Bandwidth $(\mathrm{Hz})$
}

Thermal noise voltage cannot be removed completely, but it can be reduced by decreasing the bandwidth. However, it is not possible to decrease the temperature in a large fraction of transducers and amplifiers towards absolute zero point in order to decrease the noise level. Changing the transducer resistance will only help minimally because the piezoresistive effect goes with the resistivity, and the noise with its square root.

\section{Shot Noise}

Shot noise forms due to unavoidable random statistical fluctuations of electric current. Shot noise might appear in the form of voltage noise when current flows through a resistor [2]. We avoid high current because resistors heat up and this affects the measurement itself.

Mathematically

$$
i_{n}=\sqrt{2 I_{q} B}
$$

where

$$
\begin{aligned}
& \text { I: DC current } \\
& \text { q: Electron charge } \\
& \text { B: Bandwidth in } \mathrm{Hz}
\end{aligned}
$$

\section{Flicker Noise (1/f Noise)}

Electronic systems and many other physical phenomena show an increasing noise at low frequency, typically below $100 \mathrm{~Hz}$. The noise amplitude rises with inverse frequency, hence this phenomenon is referred to as $1 / f$ noise or low-frequency noise. A number of explanations has been given [3] [4], such as critical phenomena, correlation of noise events, and memory effects. $1 / f$ noise reflects the fact that large deviations from the mean value may be very rare, but they happen. It can be observed in terms of fluctuations in current, voltage, or resistance [2].

\section{External Noise Sources}

Such noises are interferences caused by motors, computer screens, fans, and other electrical equipment. Proper shielding and filtering is required in order to remove such type of noise. These noise sources occur at power line frequency. Therefore, it is important to avoid such frequencies that are multiples of 50 $\mathrm{Hz}-60 \mathrm{~Hz}$ [2].

\section{The Noise Spectrum}

When we analyze the noise of such a resistive network, we find a typical feature as shown in Fig. 2.

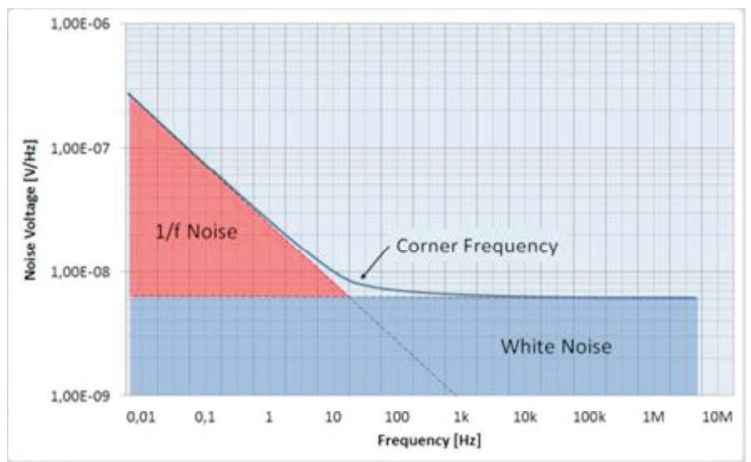

Fig. 2: Noise spectrum of a resistive sensor showing white noise and 1/f noise.

Nyquist noise is frequency-independent ("white") and spreads over the whole spectrum. $1 / f$ noise starts with a high value at $0 \mathrm{~Hz}$ and then reduces with the inverse frequency. There is a cut-off frequency, called $1 / f$ corner. Below this, 1/f noise dominates; above it, Nyquist noise dominates. For resistive sensors, the $1 / f$ corner is typically in the region of $100 \mathrm{~Hz}$. What options do we have to reduce noise? Conventional DC-excitation of the bridge will position us at the maximum of $1 / \mathrm{f}$ noise. Thus, we decide to use AC. In our experiment, we choose a measurement frequency of $f_{0}=400 \mathrm{~Hz}$, well above the $1 / \mathrm{f}$ corner. With $\mathrm{AC}$-excitation and broadband amplification, we still would integrate white noise from all frequencies, while the signal is only close to $f_{0}$. This guides us towards the next step: we need a small bandpass around $\mathrm{f}_{0}=400 \mathrm{~Hz}$. The suppression of all signals outside a small band can be performed using lock-in amplification.

\section{Lock-In Amplification}

Lock-in amplification is a technique that helps to achieve precise measurement of AC signals buried in noise. The principle of a lock-in is shown in Fig. 3. The excitation is multiplied with the output of the bridge. Then, the result is filtered by a low-pass filter. In this way, a crosscorrelation between excitation and bridge output is calculated. The true bridge signal is correlated to the excitation, and there will be an output signal. The noise is uncorrelated with the excitation, thus it cannot cause a signal after the lock-in. 
The bridge is excited by an $\mathrm{AC}$ voltage:

$V(t)=A \cos \left(\omega_{0} t\right)$

This causes a bridge output:

$V_{B}(t)=B \cos \left(\omega_{0} t+\varphi\right)+V_{N}$

The noise voltage $V_{N}$ can be considered as a superposition of many spectral terms, but we only consider one spectral term:

$V_{N}(t)=N \cos \left(\omega_{N} t\right)$

In a lock-in amplifier, the excitation is multiplied with the output:

$V_{\text {out }}(t)=$

$A \cos \left(\omega_{0} t\right) *\left[B \cos \left(\omega_{0} t+\varphi\right)+N \cos \left(\omega_{N} t\right)\right]$

using

$\cos \alpha \cos \beta=\frac{1}{2}[\cos (\alpha-\beta)+\cos (\alpha+\beta)]$.

we get

$V_{\text {out }}(t)=\frac{1}{2} A B \cos \left(2 \omega_{0} t+\varphi\right)+$

$\frac{1}{2} \mathrm{AB} \cos (\varphi)+$

$\frac{1}{2} A N \cos \left[\left(\omega_{0}+\omega_{N}\right) t\right]+$

$\frac{1}{2} A N \cos \left[\left(\omega_{0}-\omega_{N}\right) t\right]$.

The first and the third terms are quickly changing with time $\left(2 \omega_{0}\right.$ and $\omega_{0}+\omega_{N}$, respectively); this way, they will vanish after the final low pass. The second term will remain; it gives the bridge voltage to be measured. The last term vanishes for all noise components except those with a frequency close to the excitation frequency.

In this way, all noise except a small band defined by the low-pass filter is removed from the signal. As always, there is a tradeoff between noise and bandwidth: When we use a small cut-off frequency in the final low pass, then we get little noise, but the measurement bandwidth is small.

Actually, the lock-in realizes the bandpass shown in Fig. 2. Only the frequencies around $\omega_{0}$ can pass. But we used an electronic trick: it is very difficult to realize a small bandpass at a given frequency. Using the frequency mixing, we shift the bandpass to the origin. A low pass is much easier to construct as a bandpass.

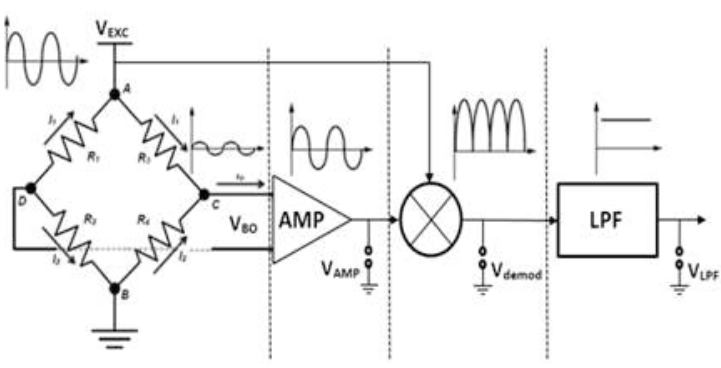

Fig. 3: Principle of lock-in amplification.

\section{Measurement Setup}

The measurement setup is based on the lock-in amplification technique. In order to analyze this technique, an experimental setup is required. Fig. 4 shows the block diagram of the experimental setup. In order to analyze the noise level at the output, a Wheatstone bridge (strain gauge load cell) is excited with an ACvoltage. A strain gauge load cell is used to convert force or load acting on it into an electrical signal. The strain gauge is attached onto a beam that deforms when force is applied on it. For temperature compensation and maximum sensitivity, four gauges are used in a full bridge configuration. The strain gauge load cell used during testing is shown in Fig. 5. The load on the load cell is applied by a flat spring. The spring is driven by a gear that is connected to a motor. The sinusoidal load on the load cell is applied by rotational motion from the motor and transversal motion by the gear. The motor speed can be varied. The rotation of the motor for the experiment was $5 \mathrm{~Hz}$.

In the circuit shown in Fig. 6, the signal from the bridge is going to the positive and negative terminals of the instrumentation amplifier, whose gain can be set with an external resistor $\left(R_{g}\right)$.

An instrumentation amplifier is used because when amplifying the $\mu \mathrm{V}$ signal coming from a transducer, severe ground loop and interference problems may arise. Amplifiers used for such applications require very low input current, drift, offset voltage, high common mode rejection, and input impedance. For such applications, instrumentation amplifiers are preferred over operational amplifiers [5].

The output of the instrumentation amplifier is connected with a demodulator. The purpose of the demodulator is to multiply the input signal (in this case, output of the instrumentation amplifier) with the carrier signal that is the reference signal. The reference signal is coming from the excitation voltage source and is fed to the demodulator chip. 
The AD630 was used because it is a balanced modulator/demodulator and because it offers a flexible range regarding accuracy and temperature stability. Output of the AD630 is either a positive or negative DC voltage because the multiplication of the input signal with the excitation signal acts as a rectification for the input signal (see Fig. 3) [7].

After modulating and demodulating the signal, it is passed through the low-pass filter, which gives a precise DC signal. An operational amplifier (OP1177) is used as a voltage follower to avoid loading the output of the low-pass filter and give a low impedance source for measuring the signal [8].

This experiment is performed with $4.8 \mathrm{~V}_{\mathrm{ac}}$ at $400 \mathrm{~Hz}$ excitation and $4.8 \mathrm{~V}_{\mathrm{dc}}$ in order to investigate the noise level in both $\mathrm{AC}$ - and $\mathrm{DC}$ excited bridge.

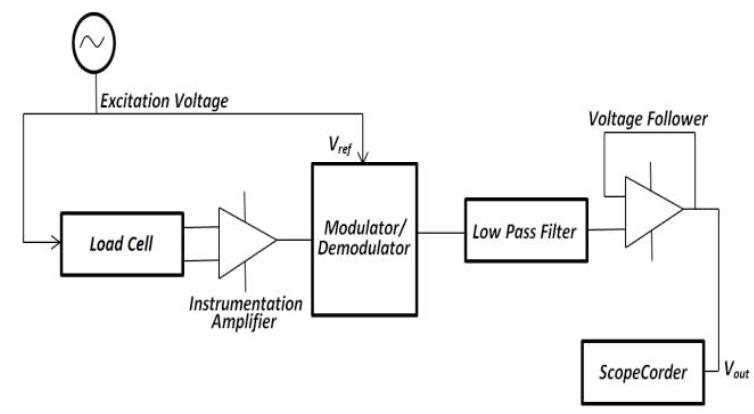

Fig. 4: Measurement setup.

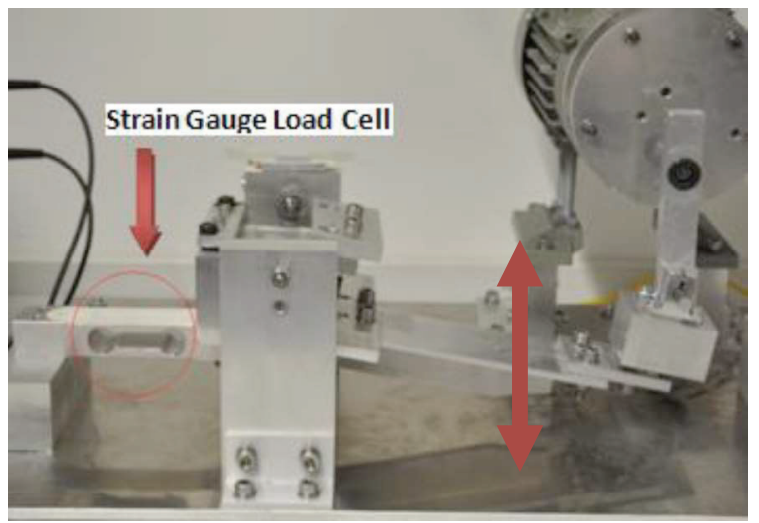

Fig. 5: Strain gauge load cell used during testing.

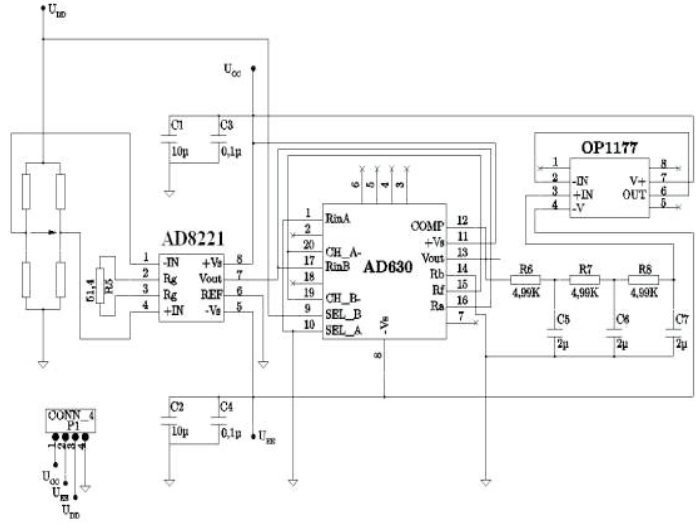

Fig. 6: Electrical circuit used to evaluate the bridge signal of the AC-driven bridge [6].

\section{Noise Factor comparison between AC- and DC-excitation}

First the bridge is excited with a DC-voltage. In this case, $4.8 \mathrm{~V}_{\mathrm{dc}}$ is applied at the strain gauge load cell excitation terminal. The amplifier's output voltage $\left(\mathrm{V}_{\mathrm{amp}}\right)$ is first measured via a scopecorder with DC-coupling of the input signal. DC-coupling allows both AC and DC components of the signal to pass through. The same procedure is repeated after selecting ACcoupling from the scopecorder. When a signal contains both $\mathrm{AC}$ and $\mathrm{DC}$ components, ACcoupling allows only $A C$ component to pass through and blocks the DC component. ACand DC-coupling is shown in Fig. 7. Then, we calculate the ratio of voltage measured with ACcoupling with respect to the DC-coupled amplifier's output voltage. This will give the noise factor when the bridge is excited with $D C$ voltage. The noise factor with DC-excitation is $500 \mathrm{mV} / \mathrm{V}$.

The same process is repeated when the bridge is excited with AC-voltage. This time, the modulator/demodulator circuit shown in Fig. 6 was used. The noise factor with AC-excitation is $9.1 \mathrm{mV} / \mathrm{V}$. This shows that the result from ACexcitation is 55 times better and that a major portion of the noise can be handled with this method.

In the next experiment, weights are placed on the load cell. It is deformed, the piezoresistors change their value, and the bridge output rises. Fig. 8 shows the noise level of the output for DC- and AC-excitation. We find again a substantial reduction of the noise level using AC-excitation and lock-in amplification. 


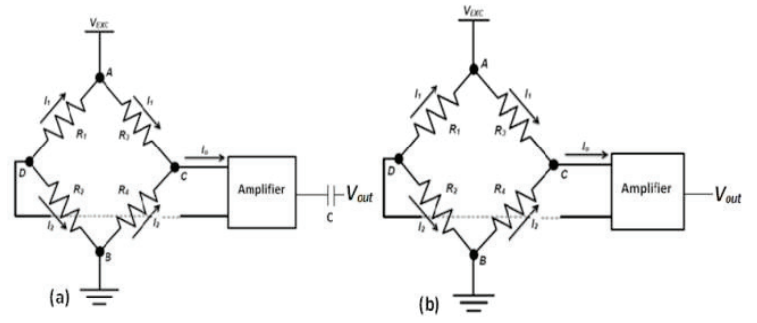

Fig. 7: (a) AC- and (b) DC- coupled signal.

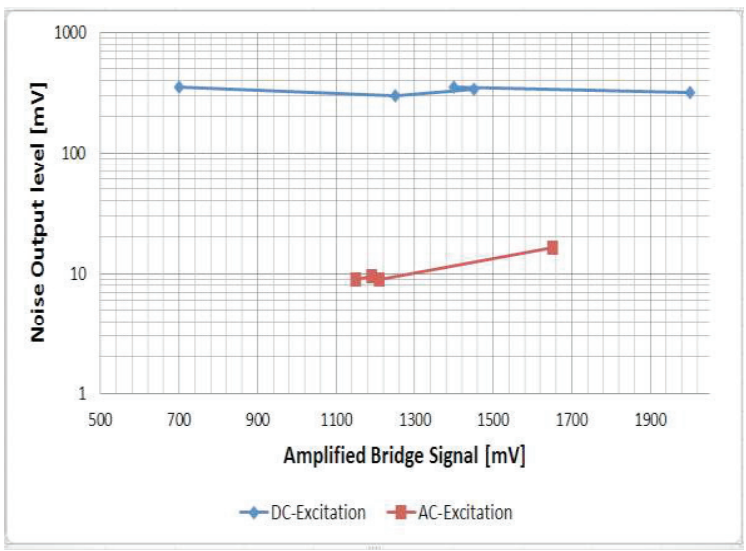

Fig. 8: Noise output using AC- and DC-excitation.

\section{Conclusion}

We investigated and compared the noise level in AC- and DC-measurement techniques of strain gauges. The strain gauge signal is very small, and an error signal might exceed the amplitude of the actual signal, thus affecting the output of the system. For high precision, it is important to measure the transducer output signal as precisely as possible without load. A number of error sources, e.g. 1/f noise, lead resistance, external sources, and thermal noise, must be considered and minimized. In order to achieve these goals, lock-in amplification, also called carrier frequency technique, and sophisticated Kelvin 4/6 wire techniques are chosen.

The proposed design is simple to operate and requires few components for its hardware implementation. The designed circuit has been tested to observe the noise reduction effect. The system can be used not only for strain gauges but everywhere where high-precision value of resistors is needed.

Results show that the noise level in ACexcitation is 55 times lower than with DCexcitation.

\section{Future Perspectives}

The proposed design shows precise measurement results. It can be used as well for multi-channel sensor systems and can be made fully autonomous. Possible applications are work piece integrated strain gauges to characterize the process of machining like CNC turning. Further work needs to investigate an increased bandwidth.

\section{References}

[1] M. Kono, T. Taura, T. Suzuki, H. Sunaga, Y. Yamada, K. Kimura, M. Morimura, H. Okano, M. Iwasaki, H. Takuno, M. Suzuki, Y. Shinoda, H. Kobayashi: A High Precision Dynamic Strain ACMeasurement Bridge Circuit. The 20th Workshop on Circuits and Systems, Karuizawa, April 23-24

[2] Application Note: About Lock-In Amplifiers, Stanford Research System.

Website www.thinkSRS.com

[3] P. Bak: Self-Organized Criticality: An Explanation of 1/f Noise. Physical Review Letters 59, 4 (1987)

[4] F. N. Hooge: 1/f Noise. Physica 83B (1976) 14-23

[5] Measurement Computing: Data Acquisition Handbook. Third Edition, Published 2004-2012 in the United States of America.

[6] M. Gerstenhaber, S. Lee: Strain Gage Measurement Using an AC Excitation, Application Note AN-683, Analog Devices. Website: http://www.analog.com/

[7] Analog Devices: AD630 Data sheet, Balanced Modulator/Demodulator. Website: http://www.analog.com/

[8] Analog Devices: OP1177 Data sheet, Precision Low Noise, Low Input Bias Current Operational Amplifiers. Website: http://www.analog.com/ 